\title{
Role of Repeated Endoscopic Ultrasound-Guided Fine Needle Aspiration for Diagnosis of Suspected Pancreatic Cancer
}

\author{
Zamani F, Haghighi $M^{*}$, Ajdarkosh H, Safarnezhad Tameshkel F, Hajibaba $M$ and \\ Motamed N \\ Gastrointestinal and Liver Diseases Research Center, Iran University of Medical Sciences, Tehran, Iran
}

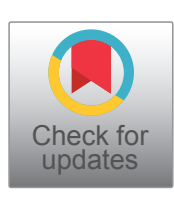

\begin{abstract}
Background \& Aims: Although Endoscopic ultrasound-guided fine needle aspiration (EUS-FNA) is useful and common method for the diagnosis of pancreatic neoplasms, the value of repeated EUS-FNA in patients with suspected pancreatic cancer but inconclusive initial EUS-FNA results is not well established.

We are going to obtain the accuracy of repeated EUS-FNA in patients with suspected pancreatic neoplasm after the inconclusive initial EUS-FNA outcome.

Methods: This is a retrospective cohort study carried out at Firoozgar hospital, a tertiary GI center.

Results: Between August 2017 and January 2020, 74 patients with repeated EUS-FNA identified and enrolled in the analysis. Final results revealed sensitivity of this method for the diagnosis of pancreatic malignancy of $83.67 \%$, specificity of $96 \%$, positive predictive value of $97.61 \%$, negative predictive value of $75 \%$ and overall accuracy of $87.83 \%$.
\end{abstract}

Conclusions: Our findings endorse that repeat EUS-FNA is a reasonable management strategy and provides reasonable accuracy.

\section{Keywords}

Endosonography, Endoscopic ultrasound-guided fine needle aspiration, Pancreatic neoplasms

\section{Introduction}

Pancreatic cancer is in the first 4 lethal cancer causing more than 30,000 deaths per year in the United States [1] As symptoms present and diagnosis process begins, pancreatic cancer is usually in advanced stages and lack of exact and accurate diagnostic methods allow its rapid progression with 1 - and 5-year-survival rates of $24 \%$ and $5 \%$, respectively [1].

Among diagnostic methods, Endoscopic ultrasound (EUS) has been shown to be the preferred imaging method for diagnosis of pancreatic cancer. It became even more accurate when provide tissue diagnosis by using fine needle aspiration [2].

The dilemma comes up when practitioner faced with clinical scenario of high probable pancreatic cancer based on clinical manifestation but a non-diagnostic pathology report of FNA tissue sample.

Despite general consensus to know EUS-FNA as the standard of care for diagnosing potential pancreatic malignancies [3], the rate of inconclusive results in the initial EUS-FNA, are quite high; ranging $6 \%-11 \%$ in different studies $[4,5]$.

Therefore, these patients remain with no definitive diagnosis despite first EUS-FNA. One possible strategy for such pa- tients is to repeat the EUS- FNA, Hence there is limited data of accuracy of repeat EUS-FNA, this method dose not generally accepted.

The aim of our study was to determine the accuracy of repeat EUS-FNA in diagnosis of suspected pancreatic cancer with inconclusive initial results to support the repeat EUSFNA strategy.

\section{Methods}

This retrospective cohort was conducted in Firoozgar hospital, Iran University of Medical Sciences. Firoozgar hospital is a tertiary referral center in Tehran province. We used collect-

*Corresponding author: Mehran Haghighi, Gastrointestinal and Liver Diseases Research Center, Iran University of Medical Sciences, Tehran, Iran, Tel: +98-9113940142, Fax: +98-2182141321

Accepted: March 11, 2021

Published online: March 13, 2021

Citation: Zamani F, Haghighi M, Ajdarkosh H, et al. (2021) Role of Repeated Endoscopic Ultrasound-Guided Fine Needle Aspiration for Diagnosis of Suspected Pancreatic Cancer. J Pancreat Cancer Treat 3(1):10-13 
Citation: Zamani F, Haghighi M, Ajdarkosh H, et al. (2021) Role of Repeated Endoscopic Ultrasound-Guided Fine Needle Aspiration for Diagnosis of Suspected Pancreatic Cancer. J Pancreat Cancer Treat 3(1):10-13

ed data in Firoozgar electronic patient registry database. All patients who underwent more than one EUS-FNA in Firoozgar hospital from August 2017 until January 2020 for evaluation of suspected pancreatic cancer with initial inconclusive result were included in this analysis.

Patients were excluded if EUS repeated without FNA or FNA was done for other reason than tissue diagnosis (e.g. amylase, tumor markers...).

Initial EUS-FNA was considered inconclusive if either sample material was inadequate/inappropriate for assessment or there was adequate sample but cytology was negative for malignancy while physician's clinical judgment was strongly suggested cancer.

Imaging characteristics of lesions leading to the first EUSFNA was documented.

All patients provided written informed consent.

EUS was performed using the linear echoendoscope (Fujinun).

All cases received moderate sedation using combination of fentanyl, propofol and midazolam. An anesthesiologist guided sedation process. The patients expended at least 2 hours after endoscopic procedure under observation in recovery room.

All FNAs were performed utilizing Echo-tip 22 French gauge EUS needle. Suction via the needle was applied in all cases. The FNA samples were evaluated visually for adequacy.

Specimens were placed on air-drying slides. They were then transported to the hospital laboratory where they were prepared and evaluated by a pathologist.

Cytology was categorized as (1) Benign/reactive, (2) Atypical, (3) Suspicious, or (4) Malignant.

The final diagnosis of pancreatic cancer was defined as one of the following criteria: (1) Report of malignancy at the second FNA, (2) Positive cytology or histology obtained by alternative means e.g. surgical biopsy, CT Scan, (3) Death from the disease within 6 months of initial EUS-FNA.

\section{Ethics}

The study was approved by the institutional review board (IRB) at Iran University of medical sciences, and all patients signed informed consent.

\section{Statistical analysis}

Continuous variables were reported as median (range) and discrete variables were expressed as $n(\%)$ unless otherwise specified. Data analysis was conducted using statistical software (SPSS Statistics_ V22, IBM, Armonk, NY, USA). Fisher's exact test was used to compare categorical variables. Student's $t$-test was used to compare continuous variables. $P$ values were calculated as 2 -tailed and a value of $\leq 0.05$ was interpreted as significant.

\section{Result}

All patients in this study were selected from Firoozgar reg- istration database between August 2017 and January 2020.

The total number of patients undergoing EUS for all indications (pancreatic masses and others) at Firoozgar hospital over the study period was 2124 . The total number of patients underwent FNA was 1398 over the study period.

The total number of patients underwent a second EUSFNA for study of a pancreatic mass over the study period was 75.

No patient met exclusion criteria. One patient was lost to follow up.

The remaining 74 patients (44 (59.5\%) male, 30 (40.5\%) female), mean age $61.35 \pm 13.63$ years (range $31-90$ ), were included in the final analysis.

The most common indication for the initial EUS was abnormal CT or MRI findings in 60 patients (81\%).

Preprocedure imaging showed suspected pancreatic mass in 56 patients $(75.7 \%)$ and dilation or stricture of biliary tree in 50 patients $(67.6 \%)$.

Cytological evaluation of the first FNA specimen was "atypical" in 5(6.75\%), "suspicious" in 15 (20.27\%), and "benign/reactive" in 54 (72.97\%).

No major complication was reported related to the first EUS.

Repeat EUS was performed in all patients after mean of 39.5 days (range 1-99 days) from the initial EUS exam.

Of 74 patients with inconclusive pathology on initial EUSFNA, 70 patients had conclusive pathology on repeat FNA (94.59\%).

Cytology was reported positive for malignancy on repeat FNA in 42 (56.8\%) patients.

The second EUS confirmed the diagnosis of cancer in 12 (60\%) out of 20 patient with prior atypical/suspicious findings at the first EUS and in $30(55.55 \%)$ out of 54 patients with benign/reactive initial cytology.

Based on the imaging characters of the lesion, malignancy was found at the second EUS-FNA in $16(63.5 \%)$ of the mass like lesion and in $24(63.2 \%)$ of the lesions with biliary tree involvement either in the form of stricture or dilation.

Analysis showed no meaningful difference between initial imaging presentation and chance of malignant diagnosis (p-value < 0.05).

Overall repeat EUS-FNA showed tissue diagnosis of malignant pancreatic lesion in 42 out of 74 patients (56.8\%). Although, one of these patients finally revealed to have benign pathology in surgical specimen.

There was no complication related to the second EUS.

Out of the 74 patients in this cohort, 49 (66.21\%) were finally confirmed to have malignancy. Repeat FNA confirmed malignancy in $41 / 49$ (83.67\%), and 6-months follow up (tissue diagnosis by other means or death related to cancer) established malignancy in 8/49 (16.32\%). 
Citation: Zamani F, Haghighi M, Ajdarkosh H, et al. (2021) Role of Repeated Endoscopic Ultrasound-Guided Fine Needle Aspiration for Diagnosis of Suspected Pancreatic Cancer. J Pancreat Cancer Treat 3(1):10-13

Table 1: Diagnostic yield of repeated EUS-FNA in patient with suspected pancreatic cancer

\begin{tabular}{|l|l|l|l|l|l|l|l|}
\hline & Year & Number of cases & Sensitivity (\%) & Specificity (\%) & PPV (\%) & NPV (\%) & Accuracy (\%) \\
\hline Eloubeidi, et al. [8] & 2008 & 24 & & & & & 84 \\
\hline Nicaud, et al. [9] & 2010 & 28 & 35 & 100 & & 61 \\
\hline Prachayakul, et al. [10] & 2012 & 8 & & & & 92.9 \\
\hline Suzuki, et al. [11] & 2013 & 84 & 95.7 & 100 & 100 & 72.7 & 96.3 \\
\hline Zhang, et al. [13] & 2016 & 43 & 62.2 & 83.3 & 95.8 & 26.3 & 65.1 \\
\hline Ávila, et al. [12] & 2016 & 34 & 61.9 & 30 & 65 & 27.3 & 51.6 \\
\hline Zamani, et al. & 2020 & 74 & 83.67 & 96 & 97.61 & 75 & 87.83 \\
\hline
\end{tabular}

According to these results, Repeat EUS-FNA providing sensitivity for the diagnosis of pancreatic malignancy of $83.67 \%$, specificity of $96 \%$, positive predictive value of $97.61 \%$, negative predictive value of $75 \%$ and overall accuracy for determination of the true final status of the patients of $87.83 \%$.

Eight of 32 patients (25\%) with non malignant second pathology underwent a third EUS-FNA at our site during the follow-up period and two of them appeared to have adenocarcinoma.

\section{Discussion}

Although EUS-FNA shows high diagnostic accuracy in patients with suspected pancreatic mass, 6 to $30 \%$ of patients with clinical and imaging findings suggestive of pancreatic cancer have inconclusive initial cytology on EUS-FNA [6,7].

Physicians face difficulties when pathology result of endosonographic aspiration is inconclusive while malignancy is highly suspicious due to clinical scenario.

The primary aim of our study was to explore how repeated FNA could facilitate access to the true diagnosis in such patient population.

Although at present, there is no universally accepted strategy on how to manage patients with cytology-clinic discrepancy, some options are available:

Patients in whom adjunctive therapy or surgery is not feasible might be followed clinically and managed supportively rather than trying to get tissue diagnosis. Even, in benign looking lesions, clinical observation and follow-up with serial imaging is possible. It is not interesting option anyway due to the risk of uncertain but potentially curable diagnosis.

CT-guided biopsy has the risk of intraperitoneal spread of the cancer via the needle track and exposure to radiation.

If the lesion appears resectable on imaging and the patient is otherwise good candidate for surgery, surgical exploration maybe considered as an option, but due to high morbidity it is not carried out solely for tissue diagnosis.

The main finding of our study is that, in patients who were suspected to have pancreatic malignancy but inconclusive initial EUS-FNA, repeat EUS-FNA was accurate in predicting the final malignant/benign status in $87.83 \%$ of the patients, sensitivity of $83.67 \%$, specificity of $96 \%$, positive predictive value of $97.61 \%$ and negative predictive value of $75 \%$.
Table 1 demonstrates the diagnostic yield of repeated EUS-FNA for patient with suspected pancreatic cancer in different studies [8-13]. Our results are shown in the last row for comparison.

Our findings showed higher accuracy than what reported before by Avila, et al., Zhang, et al., and Nicaud, et al. It was lower than what was reported by Suzuki and prachavakol, et al. though.

All together these findings endorse that repeat EUS-FNA is a reasonable management strategy.

Repeating the EUS exam and reattempting FNA is appealing option due to the minimally invasive nature of the procedure and growing evidence to support this strategy in the body of literature.

It may be some intention to get more accurate result on repeated EUS-FNA, either by endosonographer or pathologist. As it will happen in real practice too, we did not match study population for it.

Most of studies in this field had small same size and therefore they did not have enough power to evaluate for any predictors for success/failure for the repeat EUS-FNA. As EUS will become more available, there is a hope to have more comprehensive studies soon.

\section{References}

1. Jemal A, Siegel R, Ward E, et al. (2009) Cancer statistics, 2009. CA Cancer J Clin 59: 225-249.

2. Vilmann P, Saftoiu A (2006) Endoscopic ultrasound-guided fine needle aspiration biopsy: Equipment and technique. J Gastroenterol Hepatol 21: 1646-1655.

3. Eloubeidi MA, Decker GA, Chandrasekhara V, et al. (2016) The role of endoscopy in the evaluation and management of patients with solid pancreatic neoplasia. Gastrointest Endosc 83: 17-28.

4. Varadarajulu S, Tamhane A, Eloubeidi MA (2005) Yield of EUS-guided FNA of pancreatic masses in the presence or the absence of chronic pancreatitis. Gastrointest Endosc 62: 728-736.

5. Eloubeidi MA, Jhala D, Chhieng DC, et al. (2003) Yield of endoscopic ultrasound-guided fine-needle aspiration biopsy in patients with suspected pancreatic carcinoma. Cancer 99: 285-292.

6. Eloubeidi MA, Varadarajulu S, Desai S, et al. (2007) A prospective evaluation of an algorithm incorporating routine preoperative endoscopic ultrasound-guided fine needle aspiration in suspected pancreatic cancer. J Gastrointest Surg 11: 813-819. 
Citation: Zamani F, Haghighi M, Ajdarkosh H, et al. (2021) Role of Repeated Endoscopic Ultrasound-Guided Fine Needle Aspiration for Diagnosis of Suspected Pancreatic Cancer. J Pancreat Cancer Treat 3(1):10-13

7. Turner BG, Cizginer S, Agarwal D, et al. (2010) Diagnosis of pancreatic neoplasia with EUS and FNA: A report of accuracy. Gastrointest Endosc 71: 91-98.

8. Eloubeidi MA, Varadarajulu S, Desai S, et al. (2008) Value of repeat endoscopic ultrasound-guided fine needle aspiration for suspected pan-creatic cancer. J Gastroenterol Hepatol 23: 567570.

9. Nicaud M, Hou W, Collins D, et al. (2010) The utility of repeat endoscopic ultrasound-guided fine needle aspiration for suspected pancreatic cancer. Gastroenterol Res Pract 2010: 268290.

10. Prachayakul V, Sriprayoon T, Asawakul P, et al. (2012) Repeated endoscopic ultrasound guided fine needle aspiration (EUS-FNA) improved diagnostic yield of inconclusive initial cytology for sus- pected pancreatic cancer and unknown intra-abdominal lymphadenopathy. J Med Assoc Thai 95: S68-S74.

11. Suzuki R, Lee JH, Krishna SG, et al. (2013) Repeat endoscopic ultrasound-guided fine needle aspiration for solid pancreatic lesions at a tertiary referral center will alter the initial inconclusive result. J Gastrointestin Liver Dis 22: 183-187.

12. Téllez-Ávila FI, Martínez-Lozano JA, Valdovinos-Andraca F, et al. (2016) Repeat endoscopic ultrasound fine needle aspiration after a first negative procedure is useful in pancreatic lesions. Endosc Ultrasound 5: 258-262.

13. Zhang B, Zhu F, Li P, et al. (2018) Endoscopic ultrasound elastography in the diagnosis of pancreatic masses: A meta-analysis. Pancreatology 18: 833-840. 